\title{
La matemática como dominio masculino: Un estudio de la percepción en la educación media costarricense ${ }^{1}$
}

\author{
Mathematics as a Male Domain: A Study of Perception in Costa Rican Secondary Education²
}

A matemática como domínio masculino: um estudo da percepção no ensino médio da Costa Rica ${ }^{3}$

Luis Gerardo Meza-Cascante Instituto Tecnológico de Costa Rica

Escuela de Matemática

Cartago, Costa Rica

gemeza@tec.ac.cr

(iD) https://orcid.org/0000-0002-5413-0172

Zuleyka Suárez-Valdés-Ayala Instituto Tecnológico de Costa Rica

Escuela de Matemática

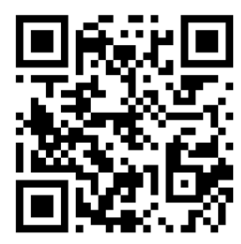

Cartago, Costa Rica

zsuarez@tec.ac.cr

iD https://orcid.org/0000-0002-1822-4825

Evelyn Agüero-Calvo Instituto Tecnológico de Costa Rica

Escuela de Matemática

Cartago, Costa Rica

evaguero@tec.ac.cr

iD https://orcid.org/0000-0002-2294-0357

Rodolfo Jiménez-Céspedes Instituto Tecnológico de Costa Rica Escuela de Ciencias Naturales y Exactas

Cartago, Costa Rica

rodjimenez@tec.ac.cr

iD https://orcid.org/0000-0002-4704-6351

\footnotetext{
- Se reportan los resultados del proyecto de investigación EDOMEM: Estudio de la matemática como "dominio masculino" en la educación media (Meza-Cascante et al., 2018), desarrollado en la Escuela de Matemática del Instituto Tecnológico de Costa Rica con el código 0144-0014.

${ }^{2}$ We report the results of the research project EDOMEM: A Study of mathematics as a "male domain" in secondary education (Meza-Cascante et al., 2018), developed at the School of Mathematics of the Technological Institute of Costa Rica with the code 0144-0014.

${ }^{3}$ São apresentados os resultados do projeto de investigação EDOMEM: estudo da matemática como "domínio masculino" no ensino médio (Meza-Cascante et al., 2018), desenvolvido na Escola de Matemática do Instituto Tecnológico de Costa Rica com o código 0144-0014.
} 
http://doi.org/10.15359/ree.25-3.35

http://www.una.ac.cr/educare

educare@una.ac.cr
Martha Calderón-Ferrey Instituto Tecnológico de Costa Rica Escuela de Ciencias Sociales

Cartago, Costa Rica micalderon@tec.ac.cr https://orcid.org/0000-0002-0571-0859

Laura Sancho-Martínez Instituto Tecnológico de Costa Rica Escuela de Cultura y Deporte Cartago, Costa Rica lasancho@tec.ac.cr https://orcid.org/0000-0002-0946-9505

Patricia Pérez-Tyteca Universidad de Alicante Departamento de Innovación y Formación Didáctica Alicante, España patricia.perez@ua.es iD https://orcid.org/0000-0002-7796-9042

Javier Monje-Parrilla Universidad de Alicante Departamento de Innovación y Formación Didáctica

Alicante, España monjejavier@ua.es https://orcid.org/0000-0003-0020-2991

Recibido • Received • Recebido: 30 / 11 / 2019

Corregido • Revised • Revisado: 23 / 07 / 2021

Aceptado • Accepted • Aprovado: 25 / 08 / 2021

\section{Resumen:}

Introducción. Se presentan los resultados de un estudio de la percepción de la matemática como dominio masculino, en estudiantes de educación media oficial diurna costarricense, realizado con la intención de evaluar la necesidad de desarrollar programas de intervención que promuevan las mismas competencias en las mujeres que los hombres en matemática. Se entiende como "dominio masculino" la creencia de que los hombres presentan mejores condiciones que las mujeres para aprender matemática y, en general, para desempeñarse en ambientes relacionados con esa disciplina. Metodología. Se trata de una investigación cuantitativa de tipo descriptivo, que estudia la variable por sexo, nivel educativo o zona de ubicación del colegio, aplicando la escala Matemática como dominio masculino de Fennema y Sherman (1976). La muestra constó de 3581 estudiantes en el año 2018 (50,8\% mujeres), y fue seleccionada por muestreo simple por conglomerados. En el análisis de los datos se utilizaron las técnicas estadísticas t de Student Ty Welch (complementada con la prueba 
de Scheffé). Resultados. Se encontraron diferencias entre hombres y mujeres: estas muestran en los hombres niveles superiores en la variable, con tamaño del efecto moderado. Se detectaron diferencias entre el estudiantado de sétimo y octavo nivel con los de noveno, décimo y undécimo, con valores superiores para los dos primeros niveles. También se encontraron diferencias entre los colegios ubicados en zonas urbanas y los de las zonas rurales; los segundos manifiestan niveles mayores de percepción de la matemática como dominio masculino, con tamaño del efecto moderado. Discusión. La investigación devela que aproximadamente un $84 \%$ del estudiantado tiene niveles de percepción de la matemática como dominio masculino entre bajos y muy bajos, lo que se aprecia como un hallazgo positivo. Los resultados sugieren la conveniencia de desarrollar tales programas de intervención.

Palabras claves: Percepción; matemáticas; educación secundaria; dominio masculino.

\begin{abstract}
:
Introduction. The results of a study on the perception of mathematics as a male domain in official day high school students in Costa Rica are presented. The study was conducted to evaluate the need to develop intervention programs that promote that women have the same capabilities as men in mathematics. "Male domain" is understood as the belief that men have better conditions than women to learn mathematics and, in general, to perform in environments related to that discipline. Method. This descriptive quantitative research studies the variable by sex, educational level, or school location area, applying the scale Mathematics as a male domain by Fennema and Sherman (1976). The sample consisted of 3,581 students in 2018 (50.8\% female), selected by simple cluster sampling. In the data analysis, the statistical techniques of Student's $t$ and Welch (complemented with the Scheffé test) were used. Results. Differences were found between men and women, with men showing higher levels in the variable, with a moderate effect size. Differences were detected between the seventh and eighth level students with those of the ninth, tenth and eleventh levels, with higher values for the first two levels. Differences were also found between schools located in urban areas and those in rural areas, showing higher levels of perception of mathematics as a male domain in the latter, with a moderate effect size. Discussion. The research reveals that approximately $84 \%$ of the students have low to very low levels of perception of mathematics as male dominance, which is seen as a positive finding. The results suggest the convenience of developing intervention programs that promote that women have the same competencies as men in mathematics.
\end{abstract}

Keywords: Perception; mathematics; secondary education; male domain.

\title{
Resumo
}

Introdução. São apresentados os resultados de um estudo sobre a percepção da matemática como domínio masculino, nos estudantes do ensino médio oficial diurno da Costa Rica, realizado com o intuito de avaliar a necessidade de desenvolver programas de intervenção que promovam que as mulheres tenham as mesmas competências que os homens na matemática. Metodologia. Tratase de uma pesquisa quantitativa descritiva que estuda a variável por sexo, escolaridade ou área de localização da escola, aplicando a escala Matemática como domínio masculino de Fennema e Sherman (1976). A amostra foi de 3.581 alunos em 2018 (50,8\% do sexo feminino), selecionados por amostragem simples por conglomerados. $\mathrm{Na}$ análise dos dados, foram utilizadas as técnicas estatísticas t de Student e Welch (complementadas com o teste de Scheffé). Resultados. Foram 
http://doi.org/10.15359/ree.25-3.35

http://www.una.ac.cr/educare

educare@una.ac.cr

\begin{abstract}
encontradas diferenças entre homens e mulheres, com os homens apresentando níveis mais elevados na variável, com tamanho de efeito moderado. Detectaram-se diferenças entre os estudantes do sétimo e oitavos níveis com os do nono, décimo e décimo primeiro níveis, com valores maiores para os dois primeiros níveis. Também foram encontradas diferenças entre as escolas localizadas na área urbana e as da área rural, mostrando níveis mais elevados de percepção da matemática como domínio masculino nesta última, com tamanho de efeito moderado. Discussão. A pesquisa revela que aproximadamente $84 \%$ dos estudantes têm níveis baixos a muito baixos de percepção da matemática como dominância masculina, o que é visto como uma descoberta positiva. Os resultados sugerem a conveniência de desenvolver programas de intervenção que promovam que as mulheres tenham as mesmas competências que os homens na matemática.
\end{abstract}

Palavras-chave: Percepção; matemática; ensino médio; domínio masculino.

\title{
Introducción
}

Como señalan Farfán Márquez y Simón Ramos (2013), tradicionalmente se ha considerado que el campo de la matemática, y otras áreas relacionadas, son de dominio masculino. El llamado "dominio masculino" en matemática alude a la creencia de que los hombres presentan mejores condiciones que las mujeres para aprender matemática y, en general, para desempeñarse en ambientes relacionados con esa disciplina.

Los resultados de investigaciones que incluyen el factor afectivo reportan la existencia de creencias generalizadas, y muy arraigadas, acerca de la menor capacidad de las mujeres para aprender y trabajar en matemáticas (Bian et al., 2017; Eccles, 1987, citado por Rodríguez Muñoz, 2011; Nurlu, 2017). Los trabajos de Burton, 1996; Parker et al., 1995; Fennema y Leder, 1990, citados por González Jiménez (2006) pusieron de manifiesto que esa visión ha restringido la participación de las mujeres como estudiantes de la disciplina, o de otras relacionadas, y limitado su desempeño como profesionales en el campo de la matemática. También se ha documentado que los estereotipos de género, como esperar resultados menos positivos en matemática para las niñas, afecta el rendimiento académico en esa asignatura (Nurlu, 2017).

León y Salazar (2014) señalan que la creencia de que las mujeres tienen menor capacidad para la matemática por razones innatas se extendió ampliamente desde hace más de un siglo. No obstante, algunas investigaciones han revelado que no existen razones para suponer una menor capacidad de parte de las mujeres para desempeñarse en esta disciplina (Servicio de Información de Noticias Científicas, 2010, citado por León y Salazar, 2014). Tales investigaciones han revelado, tal como plantean Farfán Márquez y Simón Ramos (2013, p. 1231), que"si bien las mujeres no están en desventaja académica con los hombres, diversas situaciones del entorno las llevan a desestimar sus habilidades y a desistir de elegir carreras relacionadas con esta área".

Molina Morán (2017, p.133) plantea, a partir de sus investigaciones, que los "resultados refutan que la competencia matemática sea un dominio masculino, aunque se reconoce que 
http://doi.org/10.15359/ree.25-3.35

la cultura influye en los varones a desarrollar un interés matemático. Además, la inadecuada metodología es la causante de las actitudes negativas hacia esta materia", haciendo referencia a la metodología didáctica.

En cuanto a la percepción de las personas jóvenes sobre la matemática como dominio masculino, los resultados de las investigaciones no son del todo coincidentes. Watt (2000), citado por Núñez et al. (2005), encontró que a mayor nivel educativo se manifestaba mayor tendencia hacia la apreciación de la matemática como dominio masculino. Ursini et al. (2004, citando a Forgasz, 2001) señalan que la valoración de que las mujeres tienen menor capacidad para hacer matemática dio muestras de cambio durante algunos años, pues las investigaciones encontraron que las jóvenes y los jóvenes no estaban viendo las matemáticas como un dominio esencialmente masculino.

Jimeno Pérez (2002, p. 285), reconociendo la existencia de la creencia de que la matemática es un dominio masculino, resalta su importancia por cuanto"influye en las actitudes de las chicas hacia las matemáticas, en la percepción de sus capacidades para tener éxito en esta materia, y en sus elecciones de proseguir estudios en esta materia".

Mella (2006, p. 35) sostiene que:

La diferencia entre hombres y mujeres en sus resultados en matemáticas es discutible desde todo punto de vista; basta con mencionar quizás que en los países europeos, en las mediciones hechas por la OECD, las diferencias no son estadísticamente significativas o incluso son las mujeres las que están obteniendo mejores resultados en matemáticas.

Incluso algunas investigaciones han mostrado que las diferencias en matemática entre hombres y mujeres se han venido reduciendo (Inda-Caro et al. 2010).

El estudio de la matemática como dominio masculino es importante porque, de acuerdo con Espinosa Guia (2010, p. 32):

Las concepciones que se tienen sobre el estudio de las matemáticas como "dominio masculino" se transmiten a las mujeres de manera sutil e influyen en sus decisiones para elegir ciertos cursos y ciertas carreras que involucran a las matemáticas.

La investigación, cuyos resultados se reportan en este artículo, constituye un eslabón más en el elenco de estudios desarrollados en el Instituto Tecnológico de Costa Rica en el llamado "dominio afectivo" en el campo de la educación matemática, que aporta de manera pionera resultados sobre la percepción de las personas adolescentes costarricenses sobre si la matemática es una disciplina de predominio masculino o no. Pretende evaluar la necesidad de desarrollar programas específicos enfocados en reducir la percepción de la matemática como 
http://doi.org/10.15359/ree.25-3.35

http://www.una.ac.cr/educare

educare@una.ac.cr

dominio masculino, como parte de una estrategia más amplia de estimular las vocaciones hacia las carreras STEM (ciencias, tecnología, ingeniería y matemática) dado que persisten brechas de género, tanto en matrícula como en graduación, desfavorables para las mujeres (Programa Estado de la Educación en Desarrollo Humano Sostenible, 2019).

De manera consecuente, la investigación planteó como problema determinar cuál es la percepción del estudiantado de la educación media diurna oficial costarricense sobre la matemática como dominio masculino, estudiando la existencia de diferencias por sexo, nivel educativo o tipo de zona en la que se ubica el colegio.

La búsqueda de investigaciones similares desarrolladas en Costa Rica, en bases de datos (Web of Science, Scopus, Scielo, Redalyc, Dialnet y EBSCO), en los repositorios Kimuk y RepositorioTEC, en metabuscadores (BASE y OpenDoar) y en catálogos de universidades costarricenses (Universidad de Costa Rica, Instituto Tecnológico de Costa Rica, Universidad Nacional y Universidad Estatal a Distancia), con los términos de búsqueda mathematics, male dominance y Costa Rica, y en su versión en español, no arrojó resultados que coincidan con la temática abordada en la investigación cuyos resultados se reportan en este artículo, a saber, la percepción de la matemática como dominio masculino en estudiantes de educación media. Por ello se consideran los resultados de este estudio pioneros en el ámbito costarricense.

\section{Método}

La investigación es de tipo cuantitativo, clasificable como descriptiva dentro de ese enfoque.

\section{Participantes}

La muestra estuvo conformada por 3581 estudiantes, matriculados en el año 2018 en alguno de los cinco niveles de la educación secundaria oficial diurna de la República de Costa Rica, con la distribución por sexo y nivel que se presenta en las Tablas 1 y 2.

Tabla 1: Distribución por sexo

\begin{tabular}{ccc}
\hline Sexo & Frecuencia & Porcentaje \\
\hline Hombre & 1762 & 49.2 \\
Mujer & 1819 & 50.8 \\
\hline Total & 3581 & 100 \\
\hline
\end{tabular}

Nota: Elaboración propia. 
http://doi.org/10.15359/ree.25-3.35

http://www.una.ac.cr/educare educare@una.ac.cr

Tabla 2: Distribución por nivel educativo

\begin{tabular}{ccc}
\hline Nivel & Frecuencia & Porcentaje \\
\hline $7^{\circ}$ & 784 & 21.9 \\
$8^{\circ}$ & 717 & 20.0 \\
$9^{\circ}$ & 759 & 21.2 \\
$10^{\circ}$ & 656 & 18.3 \\
$11^{\circ}$ & 665 & 18.6 \\
\hline Total & 3581 & 100 \\
\hline
\end{tabular}

Nota: Elaboración propia.

Para seleccionar los colegios que participarían en la investigación se aplicó un muestreo aleatorio simple, estratificando por la zona de ubicación (Tabla 3), y por la cantidad de estudiantes de las diferentes provincias, a partir de un listado oficial.

Tabla 3: Distribución según tipo de región en que se ubica el colegio

\begin{tabular}{lcc}
\hline Tipo de colegio & Frecuencia & Porcentaje \\
\hline Urbano & 2469 & 68.9 \\
Rural & 1112 & 31.1 \\
\hline Total & 3581 & 100 \\
\hline
\end{tabular}

Nota: Elaboración propia.

\section{Instrumento de medición}

La percepción hacia la matemática como dominio masculino fue medida mediante la escala de tipo Likert denominada matemática como dominio masculino de Fennema y Sherman (1976), integrada por 12 ítems, y que presenta cinco opciones de escogencia que van desde "totalmente en desacuerdo" a "totalmente de acuerdo", que ha sido validada en diversas investigaciones (Nortes Martínez-Artero y Nortes Checa, 2017). 
http://doi.org/10.15359/ree.25-3.35

http://www.una.ac.cr/educare

educare@una.ac.cr

\section{Procedimiento}

El instrumento se aplicó en el formato de lápiz y papel a la segunda sección de cada nivel educativo, tras una breve introducción y el señalamiento de instrucciones, en lapsos de 15 minutos por grupo, de manera que cada estudiante completara el instrumento en forma anónima y confidencial.

\section{Análisis estadístico}

La validez del instrumento se estudió calculando el índice de discriminación de los ítems, utilizando la correlación entre la puntuación de cada ítem y el valor de la suma de los otros ítems sin incluir el ítem en análisis (Lozano Fernández y de La Fuente Solana, 2015). Tales índices fueron interpretados siguiendo lo que recomiendan Lozano Fernández y de La Fuente Solana (2015) de que un índice de discriminación mayor o igual a 0.3 es apropiado.

Se analizó, además, la unidimensionalidad de la escala mediante el análisis factorial, técnica ampliamente utilizada para estos propósitos (Jiménez Alfaro y Montero Rojas, 2013), con la finalidad de evidenciar que mide fundamentalmente solo un constructo, calculando previamente el índice KMO (de adecuación muestral de Kaiser-Meyer y Olkin), evaluado según los rangos citados en Frías-Navarro y Pascual Soler (2012) y de esfericidad de Bartlett. Como criterio de unidimensionalidad se aplicó el recomendado por Carmines y Zeller (1979), citados en Burga León (2006), que propone que el primer factor explique al menos el $40 \%$ de la varianza.

La confiabilidad de la escala se analizó con la técnica denominada Alfa de Cronbach, fijando el valor de 0.80 como mínimo aceptable (Cea D'Ancona, 1999).

Como parte del análisis estadístico descriptivo de los datos se incorpora la baremación de los puntajes, siguiendo una interpretación similar a la descrita en Agüero Calvo et al. (2017).

Posteriormente, se sometieron a contraste las siguientes hipótesis:

- Hipótesis 1: No existen diferencias, según el sexo del estudiantado, en el nivel de percepción de la matemática como dominio masculino.

- Hipótesis 2: No existen diferencias, por nivel educativo, en la percepción de la matemática como dominio masculino.

- Hipótesis 3: No existen diferencias, según el tipo de región donde se ubica el colegio, en la percepción de la matemática como dominio masculino.

Las hipótesis que involucran dos categorías (sexo y zona de ubicación del colegio) se sometieron a contraste con la prueba t de Student, bajo el supuesto de normalidad con base en el teorema del límite central. Para la hipótesis relacionada con el nivel educativo se aplicó la prueba de Welch, complementada con la prueba ad hoc de Scheffé.

8

Luis Gerardo Meza-Cascante, Zuleyka Suárez-Valdés-Ayala, Evelyn Agüero-Calvo, Rodolfo Jiménez-Céspedes, Martha Calderón-Ferrey, Laura Sancho-Martínez, Patricia Pérez-Tyteca y Javier Monje-Parrilla 
http://doi.org/10.15359/ree.25-3.35

En los casos en que se detectaron diferencias significativas se calculó el "tamaño del efecto" mediante el procedimiento reseñado en Meza Cascante et al. (2019).

\section{Resultados}

\section{Sobre la subescala}

Todos los ítems alcanzaron índices de discriminación superiores al valor mínimo aceptable de 0.3 (Lozano Fernández y de La Fuente Solana, 2015), razón por la que se asume que los mismos muestran una discriminación aceptable.

El valor del estadístico Alfa de Cronbach fue de 0.827 , lo que indica, siguiendo a Cea D’Ancona (1999), que alcanza una confiabilidad adecuada.

Posteriormente, se estableció que la subescala muestra evidencia de unidimensionalidad, con valor del índice KMO de 0.883 , un valor $\mathrm{p}<0.05$ para el índice de Bartlett y un primer factor que representó más del $40 \%$ de la varianza total explicada, previa eliminación de los ítems 1 y 2 para lograr que el primer factor explicara al menos el $40 \%$ de la varianza.

\section{Clasificación del nivel de percepción de la matemática como dominio masculino}

La media de la variable "percepción de la matemática como dominio masculino" ( $\mathrm{M}=$ $18.31, \mathrm{SD}=6.88$ ) resultó significativamente menor que el valor promedio de la escala (t $(3580)=$ -101.587, p < 0.05), lo que, interpretado de manera global, indica que el estudiantado presenta un nivel de "percepción de la matemática como dominio masculino" inferior al valor promedio; esto es, presenta niveles bajos de "percepción hacia la matemática como dominio masculino".

Una clasificación de los puntajes obtenidos para la percepción de la matemática como dominio masculino se presenta en la Tabla 4.

Tabla 4: Clasificación de la percepción de la matemática como dominio masculino

\begin{tabular}{lcc} 
Categorías & Frecuencia & Porcentaje \\
\hline Muy baja & 1478 & 41.3 \\
Baja & 1530 & 42.7 \\
Moderada & 505 & 14.1 \\
Alta & 66 & 1.8 \\
Muy alta & 1 & 0.0002 \\
\hline
\end{tabular}

Nota: Elaboración propia. 
http://doi.org/10.15359/ree.25-3.35

http://www.una.ac.cr/educare

educare@una.ac.cr

Estos resultados señalan que aproximadamente un 84\% del estudiantado muestra niveles de percepción de la matemática como dominio masculino entre bajos y muy bajos.

\section{Contraste de la hipótesis 1}

En el contraste de esta hipótesis, se comparó la media de los hombres $(M=19.65, S D=7.39)$ con la media de las mujeres $(M=17.01, S D=6.06)$, encontrándose una diferencia significativa ( $t$ $(3578)=11.695, \mathrm{p}<0.05, \mathrm{~d}=0.39)$. Es decir, cabe rechazar la hipótesis nula y acoger la hipótesis alternativa que indica que existen diferencias en el nivel de "percepción de la matemática como dominio masculino" entre los hombres y las mujeres, con media superior para los hombres y un tamaño del efecto moderado.

\section{Contraste de la hipótesis 2}

Para el contraste de esta hipótesis, se compararon los valores medios de los cinco niveles educativos utilizando la prueba deWelch, dado que la prueba de Levene $(p<0.05)$ arrojó ausencia de igualdad de varianzas. Se determinó la existencia de diferencias significativas entre al menos dos de las medias de los cinco niveles $(F(1773.697)=33.168, p<0.05)$. Mediante la prueba post hoc de Scheffé se detectó en qué niveles se dan tales diferencias, resultado que arrojó que la media de percepción de la matemática como dominio masculino es significativamente mayor en el nivel de sétimo $(M=20.30, S D=7.27)$ y octavo $(M=19.26, S D=6.67)$, comparadas con los niveles de noveno $(M=17.71, S D=6.27, p<0.05)$, décimo $(M=17.11, S D=6.56, p<0.05)$ y undécimo $(M=16.81, S D=6.92), p<0.05)$, sin que se detectaran diferencias estadísticamente significativas en el valor medio de esta variable entre algunos de los otros niveles. Por tanto, se rechazó la hipótesis nula y se aceptó la existencia de diferencias significativas en el nivel de "percepción de la matemática como dominio masculino" entre los estudiantes de sétimo y octavo en comparación con los de noveno, décimo y undécimo, con un mayor valor medio en los dos primeros niveles.

\section{Contraste de la hipótesis 3}

Para el análisis de esta hipótesis, se comparó el valor medio de los colegios de la zona urbana $(M=17.35, S D=6.62)$ con la de los colegios de la zona rural $(M=20.43, S D=6.96)$, encontrándose una diferencia significativa entre ellas, con tamaño del efecto pequeño ( $\mathrm{t}$ (3578) $=-12.405, p<0.05, d=0.45)$. Es decir, se rechaza la hipótesis nula y se acepta que existen diferencias en el nivel de percepción de la matemática como dominio masculino según la zona de ubicación del colegio, con media superior para los estudiantes de los colegios de la zona rural y con tamaño del efecto moderado. 
http://doi.org/10.15359/ree.25-3.35

http://www.una.ac.cr/educare educare@una.ac.cr

\section{Discusión}

La investigación estudió la percepción de la matemática como dominio masculino, correspondiente al espectro de variables del dominio afectivo en educación matemática, con el objetivo de indagar la existencia de diferencias significativas en su valor por sexo, nivel educativo o zona en que se ubica el colegio.

Los resultados señalan que, aproximadamente, un $84 \%$ del estudiantado muestra niveles de percepción de la matemática como dominio masculino entre bajos y muy bajos, lo que se aprecia como un hallazgo positivo. La lectura positiva de este hallazgo se reafirma con el hecho de que el valor medio de la variable en la muestra es significativamente menor que la media de la escala. Estos hallazgos no eran, en principio, los esperados, porque tal como afirman diversos autores (Nosek et al., 2009; Norlu, 2017), el estereotipo "las niñas no son buenas para la matemática" tiene alta aceptación en el mundo.

A lo anterior debe señalarse la conveniencia de no obviar la paradoja de "yo no puedo, pero nosotras sí podemos". Esta paradoja indica, de acuerdo con Rodríguez Méndez et al. (2012, p. 86), que las "chicas niegan el estereotipo, pero les cuesta identificarse personalmente con estas materias". En otras palabras, el hecho de que las mujeres del estudio hayan indicado de manera mayoritaria una posición alejada de la visión de la matemática como dominio masculino no puede interpretarse ingenuamente como que están dispuestas a asumir acciones en esa disciplina o relacionadas con esta, porque, tal como señalan Rodríguez Méndez et al. (2012, p. 86), las mujeres jóvenes "no admiten la discriminación, pero se resisten a entrar en estos dominios disciplinares. Están convencidas de que las mujeres, como colectivo, son tan capaces como los hombres, pero personalmente no emprenden la carrera científico-tecnológica", lo que en el caso costarricense se plasma en las brechas de género en contra de las mujeres, tanto en matrícula como en graduación, en las carreras STEM (Programa Estado de la Educación en Desarrollo Humano Sostenible, 2019).

La diferencia en el nivel de percepción de la matemática como dominio masculino encontrada entre hombres y mujeres, con niveles promedio superiores para los hombres, tiene importancia práctica porque el tamaño del efecto resultó moderado. En consecuencia, desde una perspectiva práctica estos resultados sugieren la conveniencia de desarrollar programas de intervención en la educación formal tendientes a promover la visión de que no existen razones válidas para asumir posiciones de la matemática como dominio masculino. Es decir, las mujeres tienen las mismas competencias que los hombres en esa disciplina.

Los resultados sugieren que el estudiantado de los dos primeros niveles tiene, en promedio, una visión más conservadora de que la matemática es de domino masculino. No obstante, el resultado puede interpretarse como positivo porque implica que el estudiantado, al avanzar en sus estudios, mejora su apreciación de que tanto los hombres 
http://doi.org/10.15359/ree.25-3.35

http://www.una.ac.cr/educare

educare@una.ac.cr

como las mujeres tienen la misma competencia para la matemática. Y, desde una perspectiva práctica y optimista, podría esperarse que ese mejoramiento de la percepción de la capacidad matemática de las mujeres ayude a que estas seleccionen con mayor frecuencia carreras universitarias relacionadas con dicha ciencia. Esto es algo necesario, conveniente y oportuno en países como Costa Rica, que necesitan incrementar la formación de profesionales en las áreas STEM, a la vez que se reducen las brechas de género en la matrícula y en la graduación en carreras de ese ámbito.

Los resultados por zona de ubicación del colegio indican la existencia de diferencias, con un tamaño del efecto moderado. Este hallazgo también sugiere la necesidad de que se desarrollen acciones concretas en la educación formal, en este caso con preponderancia en los colegios ubicados en zona rural, que contribuyan a disminuir la diferencia detectada.

\section{Agradecimientos}

Se agradece el financiamiento otorgado por la Vicerrectoría de Investigación y Extensión del Instituto Tecnológico de Costa Rica para el desarrollo del estudio y a los colegios participantes en la recolección de los datos.

\section{Declaración de Material complementario}

Este artículo tiene disponible, como material complementario:

-La versión preprint del artículo en https://doi.org/10.5281/zenodo.4796776

\section{Referencias}

Agüero Calvo, E., Meza Cascante, L. G., Suárez Valdés-Ayala, Z. y Schmidt Quesada, S. (2017). Estudio de la ansiedad matemática en la educación media costarricense. Revista Electrónica de Investigación Educativa, 19(1), 35-45. https://doi.org/10.24320/redie.2017.19.1.849

Bian, L., Leslie, S.-J.y Cimpian, A. (2017). Gender stereotypes about intellectual ability emerge early and influence children's interests. Science, 355(6323), 389-391. https://doi.org/10.1126/ science.aah6524

Burga León, A. (2006). La unidimensionalidad de un instrumento de medición: Perspectiva factorial. Revista de Psicología, 24(1), 53-80. https://doi.org/10.18800/psico.200601.003

Cea D’Ancona, M. Á. (1999). Metodología cuantitativa: Estrategias y técnicas de investigación social. Síntesis Sociología. 
http://doi.org/10.15359/ree.25-3.35

Espinosa Guia, C. G. (2010). Diferencias entre hombres y mujeres en educación matemática: ¿Qué pasa en México? Investigación y Ciencia, 18(46), 28-35. https://www.redalyc.org/ articulo.oa?id $=67413508005$

Farfán Márquez, R. M. y Simón Ramos, M. G. (2013). Género y desarrollo del talento en matemáticas. En R. Flores (Ed.), Acta Latinoamericana de Matemática Educativa (Vol. 26, pp. 1231-1240). Clame. https://www.researchgate.net/publication/262105654 Genero y desarrollo del talento en matematicas

Fennema, E. y Sherman, J. A. (1976). Fennema-Sherman Mathematics Attitudes Scales: Instruments Designed to Measure Attitudes Toward the Learning of Mathematics by Females and Males. Journal for Research in Mathematics Education, 7(5), 324-326. https:// doi.org/10.5951/jresematheduc.7.5.0324

Frías-Navarro, D. y Pascual Soler, M. (2012). Prácticas del análisis factorial exploratorio (AFE) en la investigación sobre conducta del consumidor y marketing. Suma Psicológica, 19(1), 45-58. https://www.uv.es/ friasnav/FriasNavarroMarcopsSoler.pdf

González Jiménez, R. M. (2006). Mujeres matemáticas: Análisis del caso en México. Revista Cuestiones de géneros, (1), 113-135. http://revistas.unileon.es/index.php/ cuestionesdegenero/article/viewFile/3856/2731

Inda-Caro, M., Rodríguez-Menéndez, C. y Peña-Calvo, V. (2010). PISA 2006: La influencia del género en los conocimientos y competencias científicas. Revista Iberoamericana de Educación, 51(2), 1-12. https://doi.org/10.35362/rie5121836

Jiménez Alfaro, K. y Montero Rojas, E. (2013). Aplicación del modelo de Rasch, en el análisis psicométrico de una prueba de diagnóstico en matemática. Revista digital Matemática, Educación e Internet, 13(1), 1-23. https://doi.org/10.18845/rdmei.v13i1.1628

Jimeno Pérez, M. (2002). Al otro lado de las fronteras de las matemáticas escolares: Problemas y dificultades en el aprendizaje matemático de los niños y niñas de tercer ciclo de primaria [Tesis doctoral]. Universidad de Málaga. http://www.biblioteca.uma.es/bbldoc/ tesisuma/16275718.pdf

León, V. y Salazar, A. (2014). Diferencias de género en matemática y lenguaje en alumnos de colegios adventistas en el sistema de medición de la calidad de la Educación (SIMCE) en Chile. Apuntes Universitarios. Revista de Investigación, 4(2), 81-106. https://www.redalyc. org/toc.oa?id=4676\&numero $=46129$

Lozano Fernández, L. M. y de la Fuente Solana, E. (2015). Diseño y validación de cuestionarios. En A. Pantoja-Vallejo (Coord.), Manual básico para la realización de tesinas, tesis y trabajos de investigación (pp. 247-272). Editorial EOS. 
http://doi.org/10.15359/ree.25-3.35

http://www.una.ac.cr/educare

educare@una.ac.cr

Mella, O. (2006). Factores que afectan los resultados de la escuela pública chilena. REICE - Revista Electrónica Iberoamericana sobre Calidad, Eficacia y Cambio en Educación, 4(1), 29-37. https://revistas.uam.es/index.php/reice/article/view/5562/5982

Meza Cascante, L. G., Agüero Calvo, E., Suárez Valdés-Ayala, Z., Calderón Ferrey, M., Sancho Martínez, L., Jiménez Céspedes, R., Pérez Tyteca, P. y Monje Parrilla, J. (2018). EDOMEM: Estudio de la matemática como "dominio masculino" en la educación media [Informe final de proyecto de investigación, inédito]. Instituto Tecnológico de Costa Rica.

Meza Cascante, L. G., Agüero Calvo, E., Suárez Valdés-Ayala, Z., Calderón Ferrey, M., Sancho Martínez, L., Pérez Tyteca, P. y Monje Parrilla, J. (2019). Actitud hacia la matemática: Percepción de la actitud de padres. Comunicación, 28(1), 4-15. https://dx.doi.org/10.18845/ rc.v28i1-2019.4437

Molina Morán, E. (2017). Creencias y actitudes sobre género y educación matemática en la formación del profesorado de preescolar. UNION Revista Iberoamericana de Educación Matemática, 13(50), 133-152. http://www.fisem.org/www/union/revistas/2017/50/07.pdf

Nortes Martínez-Artero, R. y Nortes Checa, A. (2017). Competencia matemática, actitud y ansiedad hacia las matemáticas en futuros maestros. Revista Electrónica Interuniversitaria de Formación del Profesorado, 20(3), 145-160. http://dx.doi.org/10.6018/reifop.20.3.290841

Nosek, B. A., Smyth, F. L., Sriram, N., Lindner, N. M., Devos, T., Ayala, A., Bar-Anan, Y., Bergh, R., Caie, H., Gonsalkorale, K., Kesebir, S., Maliszewski, N., Neto, F., Olli, E., Park, J., Schnabel, K., Shiomura, K., Tulbure, B. T., Wiersn, R. W. ..., Greenwald, A. G. (2009). National differences in gender-science stereotypes predict national sex differences in science and math achievement. Proceedings of the National Academy of Sciences of the United States of America, 106(26), 10593-10597. http://dx.doi.org/10.1073/pnas.0809921106

Núñez, J. C., González-Pienda, J. A., Álvarez, L., González, P., González-Pumariega, S., Roces, C., Castejón, L., Solano, P., Bernardo, A., García, D., Da Silva, E. H., Rosário, P. y Rodríguez Feio, L. do S. (2005). Las actitudes hacia las matemáticas: Perspectiva evolutiva. Universidad de Oviedo. https://www.educacion.udc.es/grupos/gipdae/documentos/congreso/ viiicongreso/pdfs/291.pdf

Nurlu, Ö. (2017). Developing a teachers' gender stereotype scale toward mathematics. lejee. Intenational Electronic Journal of Elementary Education, 10(2), 287-299. https://doi. org/10.26822/iejee.2017236124 
http://doi.org/10.15359/ree.25-3.35 http://www.una.ac.cr/educare educare@una.ac.cr

Programa Estado de la Educación en Desarrollo Humano Sostenible (2019). Resumen séptimo informe estado de la educación (7.. ed.). PEN. https://estadonacion.or.cr/wp-content/ uploads/2019/08/Estado-Educacio\%CC\%81n-RESUMEN-2019-WEB.pdf

Rodríguez Méndez, M. del C., Peña Calvo, J. V. e Inda Cxaro, M. (2012). Creencias de autoeficacia y elección femenina de estudios científico-tecnológicos: Una revisión teórica de su relación. Teoría de la Educación. Revista Interuniversitaria, 24(1), 81-104. https://doi. org/10.14201/10333

Rodríguez Muñoz, C. (2011). Mujeres y matemáticas escolares: Construcción de representaciones sociales. Memorias de la 13 Conferencia Interamericana de Educación Matemática CIAEM, Recife, Brasil. https://ciaem-redumate.org/ocs/index.php/xiii ciaem/xiii ciaem/paper/ viewFile/2375/713

Ursini, S., Sánchez, G., Orendain, M. y Butto, C. (2004). El uso de la tecnología en el aula de matemáticas: Diferencias de género desde la perspectiva de los docentes. Enseñanza de las Ciencias, 22(3), 409-424. https://www.raco.cat/index.php/Ensenanza/article/ download/21991/21825 